ARTICLE

DOI: $10.1038 / s 41467-018-04354-x$

\title{
k-space imaging of anisotropic 2D electron gas in GaN/GaAIN high-electron-mobility transistor heterostructures
}

L.L. Lev ${ }^{1,2}$, I.O. Maiboroda ${ }^{2}$, M.-A. Husanu1,3, E.S. Grichuk², N.K. Chumakov², I.S. Ezubchenko², I.A. Chernykh², X. Wang ${ }^{1}$, B. Tobler ${ }^{1}$, T. Schmitt', M.L. Zanaveskin ${ }^{2}$, V.G. Valeyev (1) ${ }^{2}$ \& V.N. Strocov ${ }^{1}$

Nanostructures based on buried interfaces and heterostructures are at the heart of modern semiconductor electronics as well as future devices utilizing spintronics, multiferroics, topological effects, and other novel operational principles. Knowledge of electronic structure of these systems resolved in electron momentum $k$ delivers unprecedented insights into their physics. Here we explore 2D electron gas formed in GaN/AIGaN high-electron-mobility transistor heterostructures with an ultrathin barrier layer, key elements in current highfrequency and high-power electronics. Its electronic structure is accessed with angleresolved photoelectron spectroscopy whose probing depth is pushed to a few nanometers using soft-X-ray synchrotron radiation. The experiment yields direct $\mathbf{k}$-space images of the electronic structure fundamentals of this system-the Fermi surface, band dispersions and occupancy, and the Fourier composition of wavefunctions encoded in the k-dependent photoemission intensity. We discover significant planar anisotropy of the electron Fermi surface and effective mass connected with relaxation of the interfacial atomic positions, which translates into nonlinear (high-field) transport properties of the GaN/AIGaN heterostructures as an anisotropy of the saturation drift velocity of the $2 \mathrm{D}$ electrons.

\footnotetext{
${ }^{1}$ Swiss Light Source, Paul Scherrer Institute, 5232 Villigen-PSI, Switzerland. 2 National Research Centre "Kurchatov Institute", 123182 Moscow, Russia.

${ }^{3}$ National Institute of Materials Physics, Atomistilor 405A, 077125 Magurele, Romania. These authors contributed equally: L. L. Lev, I. O. Maiboroda, V. N.

Strocov. Correspondence and requests for materials should be addressed to V.N.S. (email: vladimir.strocov@psi.ch)
} 
T he concept of high-electron-mobility transistors (HEMTs) advanced by Mimura ${ }^{1}$ in the early 80 s for GaAs/GaAlAs heterostructures revolutionized the field of high-frequency semiconductor electronics. It exploits an idea of polarization engineering when a large band offset between an intrinsic semiconductor and a doped barrier layer forms a quantum well (QW) at the interface that confines a mobile two-dimensional electron gas (2DEG) on the intrinsic semiconductor side. Its spatial separation from defects in the doped barrier layer and in the interface region-in contrast to conventional transistor structures where the $2 \mathrm{DEG}$ is formed by doping-allows the electrons to escape defect scattering and dramatically increase their mobility $\mu_{\mathrm{e}}$, limited then only by phonon scattering. This fundamental operational principle of HEMTs boosts their high-frequency performance, which is exploited in a wide spectrum of applications such as cell phones.

A characteristic property of HEMTs based on wurtzite GaN/ AlGaN heterostructures is the accumulation of large sheet carrier concentrations $n_{\mathrm{s}} \sim 10^{13} \mathrm{~cm}^{-2}$-about one order of magnitude higher compared with other III-V or elementary semiconductors $^{2,3}$ - without intentional doping of the barrier layer. This property is attributed to the formation of a deep spikeshaped QW at the heterojunction, where a large conduction band offset coexists with large piezoelectric and spontaneous polarization $^{4,5}$. Although $\mu_{\mathrm{e}}$ in GaN-HEMTs is limited by a relatively large electron effective mass $m^{*} \sim 0.22$ in bulk GaN, nearly three times larger than in GaAs, these devices demonstrate an advantageous combination of sufficiently high operating frequency with exceptionally high current density, resulting from the large $n_{\mathrm{s}}$, saturation drift velocity, operating temperature, and breakdown voltage. These advantages make the GaN-HEMTs indispensable components of high-power amplifiers for microwave communication and radar systems. Recently, the ideas of creating mobile 2DEGs using spontaneous and strain-induced polarization at the interface have been extended to oxide systems such as the binary $\mathrm{Mg}_{x} \mathrm{Zn}_{1-x} \mathrm{O} / \mathrm{ZnO},{ }^{6} \mathrm{LaAlO}_{3} / \mathrm{SrTiO}_{3}{ }^{7}$, and $\mathrm{CaZrO}_{3} / \mathrm{SrTiO}_{3}{ }^{8}$ heterostructures that typically embed orders of magnitude larger $n_{\mathrm{s}}$.

The state-of-art GaN-HEMTs operate nowadays at the edge of their physical limitations, which remain far from complete understanding. Development of new strategies to improve their performance and conquer the near- $\mathrm{THz}$ operational range ${ }^{9}$ needs qualitatively new experimental knowledge about the physics of these devices. Particularly important is $\mathbf{k}$-space information about the Fermi surface (FS), band dispersions, and wavefunctions of the embedded 2DEG. These fundamental electronic structure characteristics, only indirectly accessible in optics and magnetotransport experiments such as the Hall effect, Shubnikov-de Haas oscillations, cyclotron resonance, etc. ${ }^{10-12}$, can be directly probed using the $\mathbf{k}$-resolving technique of angle-resolved photoelectron spectroscopy (ARPES). However, the small photoelectron mean free path $\lambda_{\mathrm{PE}}$ in conventional ARPES with photon energies $h v$ around $100 \mathrm{eV}$ limits its depth sensitivity to $\sim 0.5 \mathrm{~nm}$. Access to buried electron systems such as the HEMTs requires pushing this technique to the soft-X-ray energy range (SX-ARPES, see a recent review ref. 13) with $h v$ around $1 \mathrm{keV}$ and higher, where $\lambda_{\mathrm{PE}}$ grows with photoelectron kinetic energy as $\sim E_{\mathrm{k}}{ }^{3 / 4} 14,15$. For GaN in particular, elastic-peak electron spectroscopy measurements ${ }^{16}$ show that an increase of $E_{\mathrm{k}}$ from $200 \mathrm{eV}$ to $2 \mathrm{keV}$ results in an increase of $\lambda_{\mathrm{PE}}$ from $\sim 0.5$ to $4 \mathrm{~nm}$. An added virtue of SX-ARPES, still largely overlooked in applications to $2 \mathrm{D}$ systems such as QW states (QWSs), is a significantly sharper definition of the out-ofplane component $K_{z}$ of the final-state momentum $\mathbf{K}$. This fact results from the larger $\lambda_{\mathrm{PE}}$ translating, via the Heisenberg uncertainty principle, to a sharper definition of $K_{z}^{17,18}$. As we will see below, in this case the ARPES signal provides the Fourier composition of the 2DEG wavefunctions. SX-ARPES on buried systems, challenged by photoelectron attenuation in the overlayers as well as a progressive reduction of photoexcitation crosssection of the valence band (VB) states with $h v^{19}$, requires advanced synchrotron radiation sources delivering high photon flux (see Methods).

On the sample fabrication side, the 2DEG in GaN-HEMT heterostructures has until recently remained inaccessible to SXARPES because of the prohibitively large-of the order of 20-30 $\mathrm{nm}$-depth of the AlGaN barrier layers. However, recent progress in molecular beam epitaxy (MBE) technology, in pursuit of yet higher operation frequencies of these devices, has allowed fabrication of heterostructures with ultrathin barrier layers of 3-4 $\mathrm{nm}^{20-22}$, which make them ideally suited to SX-ARPES. This has allowed direct $\mathbf{k}$-space imaging of the fundamental electronic structure characteristics-the FS, electron dispersions, and the Fourier composition of wavefunctions-of the interfacial 2DEG in such heterostructures.

\section{Results}

Fabrication and basic electronic properties of GaN-HEMT heterostructures. Our samples were grown on $c$-oriented sapphire substrate (see Methods). The $500 \mathrm{~nm}$-thick Ga-polar GaN layer was grown on top of an $\mathrm{AlGaN}$ buffer layer required to suppress the crystal defects and promote growth of a smooth uniform film ${ }^{23}$. The GaN layer was overgrown by a barrier layer consisting of $2 \mathrm{~nm}$ of AlN and $1 \mathrm{~nm}$ of $\mathrm{Al}_{0.5} \mathrm{Ga}_{0.5} \mathrm{~N}$, see Fig. 1a. Hall-effect measurements on our samples, $1 \mathrm{~b}$, have found $n_{\mathrm{s}} \sim$ $8.2 \times 10^{12} \mathrm{~cm}^{-2}$ almost constant through the temperature range $T=5-300 \mathrm{~K}$ that confirms the high quality of the fabricated structures.

A simplified electronic structure model of our GaN-HEMTs is shown in Fig. 1b. It was evaluated within the conventional envelope function approach (neglecting atomic corrugation) by self-consistent solutions of the one-dimensional (1D) Poisson-Schrödinger equations with the Dirichlet boundary conditions adjusted to reproduce the experimental $n_{\mathrm{s}}$ (for details see Supplementary Note 1). The effective 1D interfacial potential $V(z)$ as a function of out-of-plane coordinate $z$ confines two QWSs with different spatial localization. The $\mathrm{QWS}_{1}$ embeds larger partial $n_{\mathrm{s}}$ and is localized closer to the interface compared to the $\mathrm{QWS}_{2}$, which is shifted into the $V(z)$ saturation region.

FS: anisotropy and carrier concentration. A scheme of our SXARPES experiment is presented in Fig. 1a. The data were acquired in the $h v$ region between 800 and $1300 \mathrm{eV}$ where the interplay of photoelectron transmission through the barrier overlayer increasing with $h v$ and photoexcitation cross-section decreasing with $h v$ maximizes the QWS signal. In view of the even QWS wavefunction symmetry, we used $p$-polarization of the incident $\mathrm{X}$-rays, which minimizes the geometry- and polarization-related matrix element effects, which distort the direct relation between ARPES intensities and Fourier composition of the QWS wavefunctions (see below).

The experimental FS map measured as a function of in-plane momentum $\mathbf{K}_{x y}=\left(K_{x}, K_{y}\right)$ at $h v=1057 \mathrm{eV}$ (maximizing the QWS signal, see below) is presented in Fig. 2a. The FS formed by the QWSs in the HEMT channel appears as tiny circles whose $\mathbf{k}_{/ /}$ are located around the $\bar{\Gamma}$-points of the heterostructure's 2D Brillouin zone (BZ) shown in Fig. 1c. The location of the FS pockets coincides with the VB maxima (VBM), as seen in an iso$E_{\mathrm{B}}$ map of the VB shown in Fig. 2b. In the direct band gap GaN, this location in $\mathbf{k}_{/ /}$is consistent with the conduction band minimum (CBM)-derived character of the QWSs.

A high-resolution cut of the FS in Fig. 2c displays an external contour with a diameter of $\sim 0.2 \AA^{-1}$ manifesting the $\mathrm{QWS}_{1}$ and 


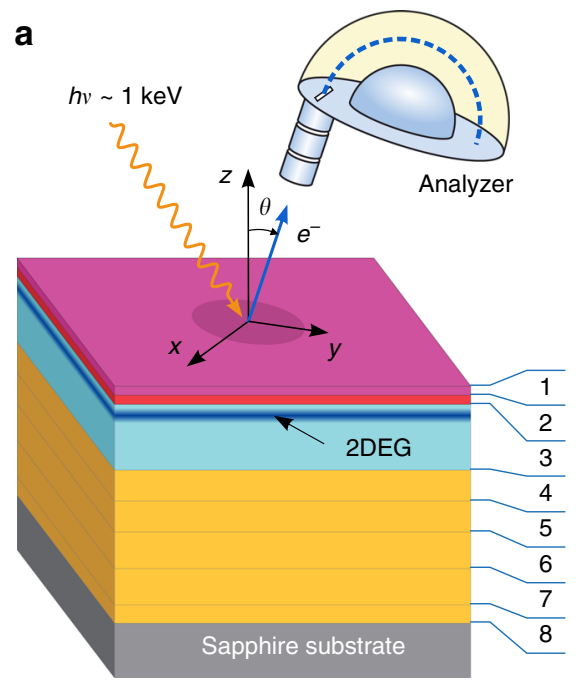

d

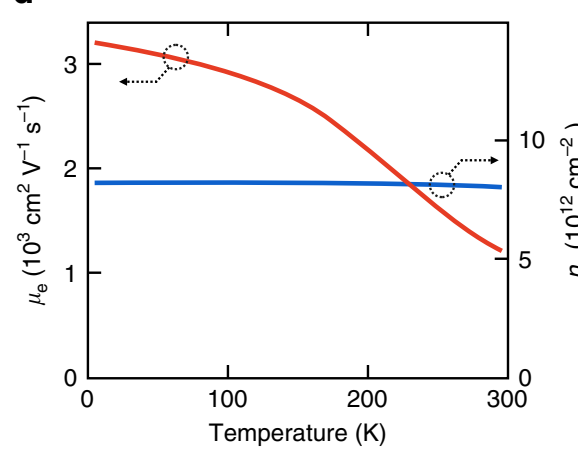

C

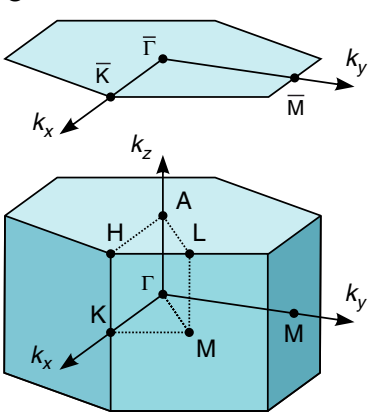

b

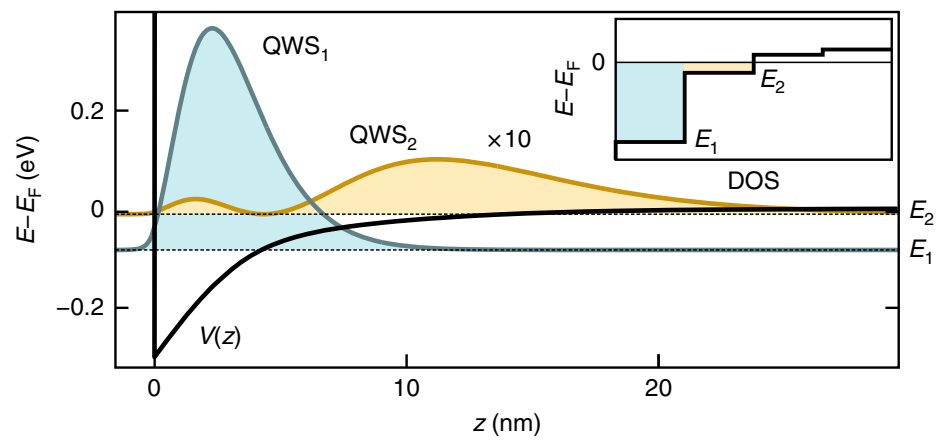

Fig. 1 SX-ARPES experiment on the GaN-HEMT heterostructure. a Scheme of the epitaxial GaN-HEMT samples investigated by SX-ARPES. The photoelectron analyser detects the distribution $I_{\mathrm{PE}}\left(E_{\mathrm{k}}, \vartheta\right)$ of the photoelectron kinetic energy $E_{\mathrm{k}}$ and emission angle $\vartheta$, which yield the binding energy $E_{\mathrm{B}}$ and momentum $\mathbf{k}$ back in the sample (corrected for the photon momentum $p=h v / c$ ) to produce the sought-for electron dispersions $E(\mathbf{k})$. $\mathbf{b} T$-dependence of $n_{\mathrm{s}}$ and $\mu_{e}$ obtained from Hall measurements. c Sketch of the electronic structure based on self-consistent solution of the 1D Poisson-Schrodinger equation. The quasi-triangular 1D potential $V(z)$ confines two QWSs having different spatial localization of their electron density $n^{i}(z)$ (exaggerated by $\times 10$ for the $\mathrm{QWS}_{2}$ ) centered at $\sim 3$ and $\sim 12 \mathrm{~nm}$ for $\mathrm{QWS}_{1}$ and $\mathrm{QWS}_{2}$, respectively. The total three-dimensional DOS (insert) show steps characteristic of the 2D states. d Bulk BZ of GaN and 2D one of the GaN/AIGaN heterostructure

finite spectral weight in the middle, suggesting the presence of $\mathrm{QWS}_{2}$. The latter is confirmed by the momentum-distribution curves (MDCs) of the Fermi intensity around the $\bar{\Gamma}_{10}$-points (Fig. 2d) where a weak structure in the middle signals the $\mathrm{QWS}_{2}$. Our observation of $\mathrm{QWS}_{2}$ is consistent with its recent detection via magnetotransport spectroscopy ${ }^{24}$. The presence of this state centered further away from the interface compared with the $\mathrm{QWS}_{1}$ indicates that $V(z)$ in the GaN-HEMTs has a long-range saturated shape (see the model in Fig. 1b). The external Fermi intensity peaks in the $E_{\mathrm{F}}$-MDCs directly identify the Fermi momenta $k_{\mathrm{F}}$ of the $\mathrm{QWS}_{1}$.

Such a comprehensive k-space view of the buried QWSs, achieved with SX-ARPES, delivers two important observations. First, the experimental $E_{\mathrm{F}}$-MDCs reveal significant anisotropy of the FS characterized by a difference of the $k_{\mathrm{F}}$ values of $0.095 \pm$ $0.006 \AA^{-1}$ along the $\overline{\Gamma M}$ azimuth and $0.085 \pm 0.004 \AA^{-1}$ along $\overline{\Gamma K}$. These values were determined from the maximal gradient of the Fermi intensity ${ }^{25}$, which delivers accurate $k_{\mathrm{F}}$-values even when the bandwidth is comparable with the experimental resolution. The indicated statistical errors are the standard deviations of the $k_{\mathrm{F}}$ values over the measurement series and scaled with the Student's $t$-distribution coefficients for a confidence interval of 76\%. The corresponding $k_{\mathrm{F}}$ anisotropy factor $A_{\mathrm{F}}=\frac{k_{\mathrm{F}}^{\bar{\Gamma}}-k_{\mathrm{F}}^{\overline{\Gamma M}}}{\left\langle k_{\mathrm{F}}\right\rangle}$, where $\left\langle k_{\mathrm{F}}\right\rangle=\left(k_{\mathrm{F}}^{\overline{\mathrm{KK}}}+k_{\mathrm{F}}^{\overline{\mathrm{MM}^{2}}}\right) / 2$, measures in our case $\sim 11 \%$. Such a considerable FS anisotropy has been completely overlooked in previous macroscopic experimental studies without k-resolution.
Why does the 2DEG show such a pronounced planar anisotropy? Our simulations of electronic structure of the GaNHEMTs (see Methods) used the standard density-functional theory (DFT), which is known to well describe excitation energies in GaN apart from the electron exchange-correlation discontinuity across the band gap approximated by the so-called scissors operator $^{26}$. As the QWSs inherit their wavefunctions from the CBM of the bulk GaN (see below), we have started our analysis from band structure. The corresponding $A_{\mathrm{F}}$ in Fig. 2e is however negligible through the whole range of $<k_{\mathrm{F}}>$ defining the band filling. Next, we approached the electronic structure of our heterostructure system using GaN/AlN slab calculations with the unit cell (u.c.) shown in Fig. 2g. The atomic positions were relaxed under the constraint of the bulk $\mathrm{GaN}$ lateral lattice constants and symmetry. The resulting $c$-oriented Ga-N bond length $d_{\mathrm{v}}$ in Fig. $2 \mathrm{f}$ shows a significant increase toward the interface relative to the bulk value. The corresponding atomic displacement contributes to the piezoelectric polarization at the GaN/AlN interface. The layer-resolved electronic structure of this system was characterized by the $\mathbf{k}_{/ /}$-resolved layer density of states (LDOS) defined as, $\rho_{z}\left(\mathbf{k}_{/ /}, E\right)=\int_{\Omega} \mathrm{d} x \mathrm{~d} y \sum_{n}\left|\psi_{n}\left(\mathbf{r}, \mathbf{k}_{/ /}, E\right)\right|^{2}$, where $\mathbf{r}=(x, y, z)$, the summation includes all $n$-th electron states with wavefunctions $\psi_{n}$ available for given $\mathbf{k}_{/ /}$and $E$, and the integration runs over the lateral unit cell $\Omega$. Figure $2 \mathrm{~h}$ shows the LDOS calculated near the CBM (the VB results are given in Supplementary Note 2) for the interface, sub-interface, and deep bulk-like GaN layers, where the bottom of the LDOS continuum corresponds to the $\mathrm{QWS}_{1}$. The corresponding $A_{\mathrm{F}}$ plots in $2 \mathrm{e}$ now 
a

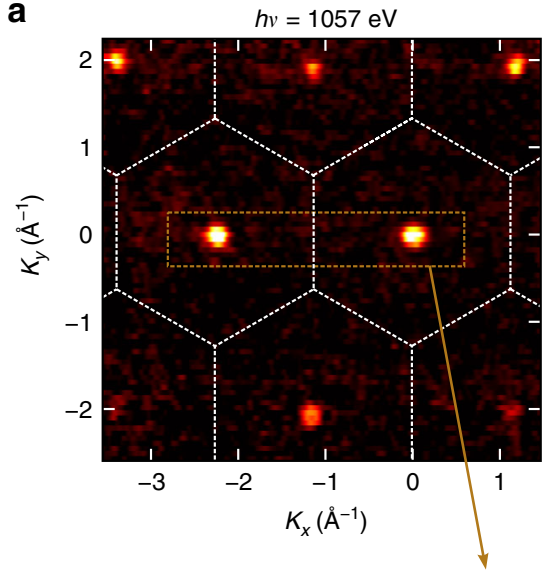

b

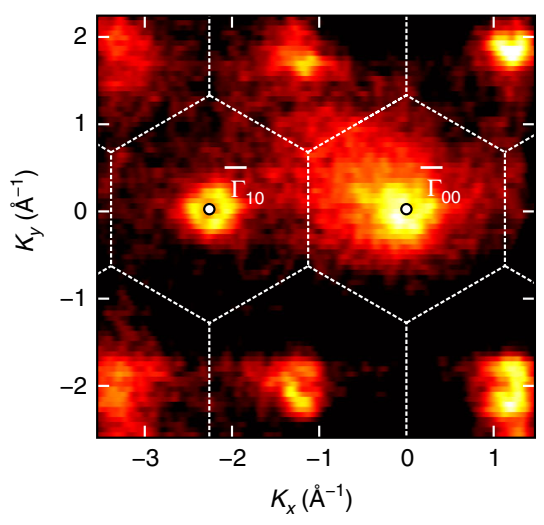

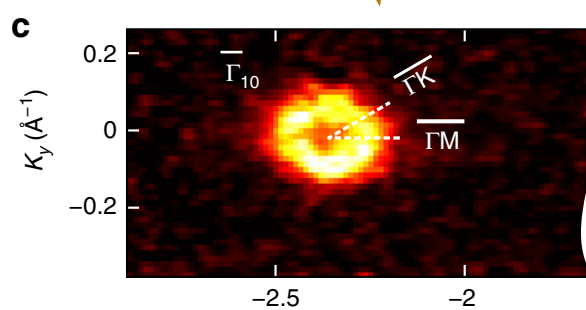
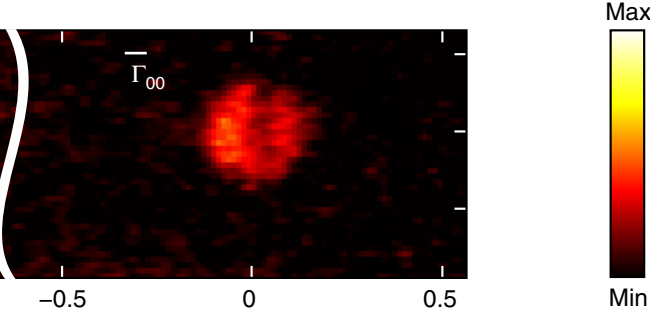

$K_{x}\left(\AA^{-1}\right)$

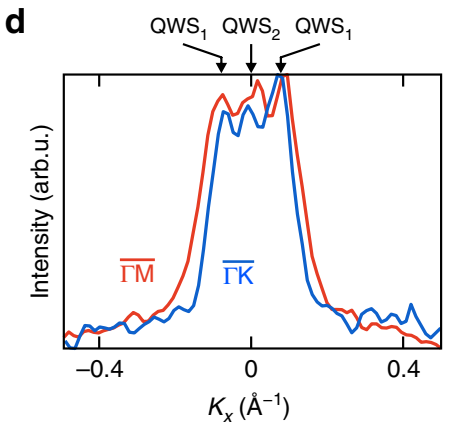

e

$\mathbf{f}$
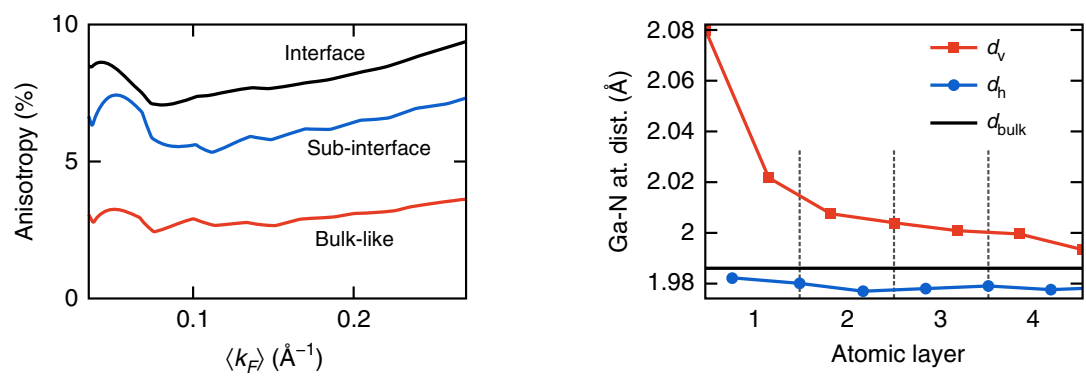

g
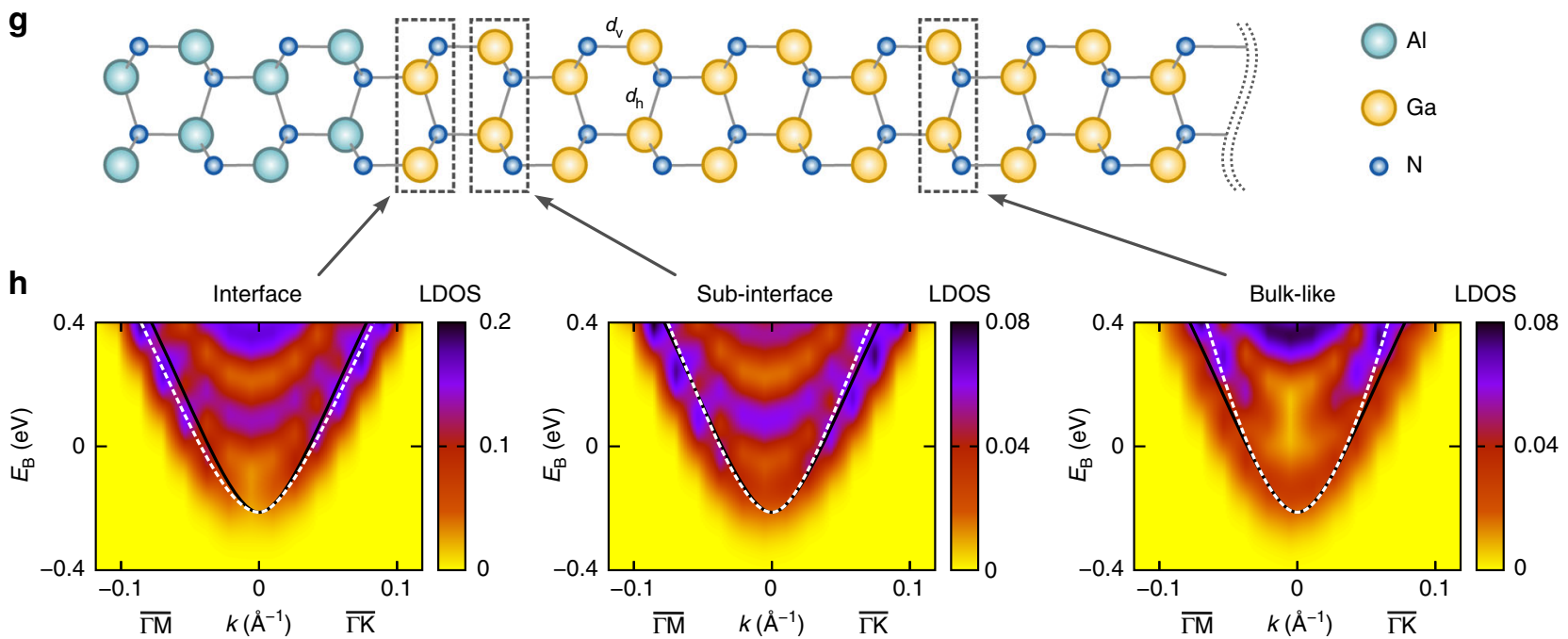

Fig. $2 \mathrm{FS}$ formed by the buried 2DEG. $\mathbf{a}$ Experimental FS formed by the 2DEG in comparison with $\mathbf{b}$ iso- $E_{\mathrm{B}}$ surface of the VB near the VBM. The FS appears as narrow electron pockets centered at the around the $\bar{\Gamma}$-points, consistent with the CBM-derived character of the 2DEG. Both VB and FS maps reflect the $C_{6 V}$ symmetry of the GaN crystal lattice. c FS along the $\bar{\Gamma}_{00}-\bar{\Gamma}_{10}$ line acquired with high energy and angle resolution. $\mathbf{d}$ MDCs of the Fermi intensity around the $\bar{\Gamma}_{10}$-point (derived from the high-statistics data in Fig. 4) identifying the tiny $\mathrm{QWS}_{2}$ and anisotropy of the QWS between the $\overline{\Gamma M}$ and $\overline{\Gamma K}$ azimuths with $A_{F} \sim$ $12 \%$. e Calculated $A_{F}$ of QWS 1 between $\overline{\Gamma M}$ and $\overline{\Gamma K}$ as a function of band filling characterized by $\left\langle k_{F}>\right.$, for bulk GaN and for various heterostructure layers. $\mathbf{f}$ Relaxation of the Ga-N bond length as a function of depth and $\mathbf{g}$ u.c. used in the slab calculations. $\mathbf{h} \mathbf{k} / /$-resolved LDOS for various heterostructure layers near the CBM with $E_{\mathrm{F}}$ adjusted to the experimental $<k_{\mathrm{F}}>$ and superimposed with the corresponding bulk $E(\mathbf{k})$ along $\Gamma M$ and $\Gamma K$ (black lines). The QWS $\mathrm{S}_{1}$ dispersion (white dashed in the bottom of the LDOS continuum) in the top GaN layers shows an asymmetry related to the interfacial atomic relaxation 
show significant anisotropy, increasing toward the interface. At the experimental $<k_{\mathrm{F}}>$ the interface layer $A_{\mathrm{F}}$ is $\sim 7 \%$, which falls almost within the error bars of the experimental value. Finally, we performed the same LDOS calculations with the atomic coordinates in the slab fixed at the bulk values (without relaxation). $A_{\mathrm{F}}$ immediately returned to the negligible bulk values. This analysis suggests thus that the discovered 2DEG anisotropy in GaN-HEMTs is a purely interface effect caused by relaxation of atomic position near the GaN/AlN interface.

We note however that the predicted atomic relaxation is restricted to a few atomic layers next to the interface, and it is not clear why it should significantly affect the 2DEG, whose maximal density is located $\sim 3 \mathrm{~nm}$ away from this region (Fig. 1b). In fact, even the state-of-art growth methods leave significant intermixing of $\mathrm{Ga}$ and $\mathrm{Al}$ atoms at the GaN/AlN interface ${ }^{21}$, resulting in a gradual variation of the lattice parameters over 1-3 $\mathrm{nm}$ from the interface. In the spirit of the entanglement between atomic relaxation and LDOS anisotropy, revealed by our computational analysis, this lattice distortion may cause significant electronic structure anisotropy extending into the 2DEG localization region.

Another important characteristic of the buried 2DEG is the experimental FS area which, by the Luttinger theorem ${ }^{27}$, is directly related to the $n_{\mathrm{s}}$ sheet carrier concentration. In our case, the area of the external QWS 1 contour translates into partial $n_{\mathrm{s}}^{(1)}=(12.8$ $\pm 1.4) \times 10^{12} \mathrm{~cm}^{-2}$, and that of the internal QWS 2 contour into $n_{\mathrm{s}}^{(2)}=(0.5 \pm 0.4) \times 10^{12} \mathrm{~cm}^{-2}$. We note that the emergence of two QWSs goes together with large $n_{\mathrm{s}}$ formed in the anomalously deep QW of the GaN-HEMTs. In our case, the $\mathrm{QWS}_{2}$ contributes only $\sim 4 \%$ of the total $n_{\mathrm{s}}$ dominated by the $\mathrm{QWS}_{1}$. A significant difference of this ratio to that of $17 \%$ found in a cyclotron resonance study ${ }^{28}$ is explained by extremely high sensitivity of the $\mathrm{QWS}_{2}$ population to the interfacial QW depth in different samples. The total $n_{\mathrm{s}}$ in our case amounts to $(13.3 \pm 1.8) \times 10^{12}$ $\mathrm{cm}^{-2}$, which is in fair agreement with $n_{\mathrm{s}}=8.2 \times 10^{12} \mathrm{~cm}^{-2}$ obtained by our Hall characterization, see Fig. 1d, in particular taking into account a small systematic overestimate of $k_{\mathrm{F}}$ introduced by the gradient method ${ }^{25}$.

Momentum dependence of ARPES intensity: wavefunction character. Within the one-step theory of photoemission-see, e.g., ref. 29-the ARPES intensity is found as $I_{\mathrm{PE}} \propto|\langle f|\mathbf{A} \cdot \mathbf{p}| i\rangle|^{2}$, where $\langle f|$ is the final and $|i\rangle$ the initial states coupled through the vector potential $\mathbf{A}$ of the incident electromagnetic field and momentum operator p. Neglecting the experimental geometry and polarization effects, this expression simplifies to the scalar product $I_{\mathrm{PE}} \propto|\langle f \mid i\rangle|^{2}$. For sufficiently high photon energies, $\langle f|$ approximates a plane wave $e^{i \mathbf{K r}}$ periodic in the in-plane $x y$ direction and damped in the out-of-plane direction $z$, and the ARPES intensity appears ${ }^{30,31}$ as $I_{\mathrm{PE}} \propto\left|\left\langle e^{i \mathbf{K r}} \mid i\right\rangle\right|^{2}$. We will now apply this formalism to the QWS wavefunctions $\psi_{\mathrm{QWS}}$.

We will first analyze $I_{\mathrm{PE}}$ as a function of photoelectron $\mathbf{K}_{x y}$ inplane momentum. If we represent $\psi_{\mathrm{QWS}}$ by Fourier expansion over $2 \mathrm{D}$ reciprocal vectors $\mathbf{g}$ as $\psi_{\mathrm{QWS}}(\mathbf{r})=\sum_{\mathbf{g}} A_{\mathbf{k}_{x y}+\mathbf{g}} e^{i\left(\mathbf{k}_{x y}+\mathbf{g}\right) \mathbf{r}_{x y}}$, the plane-wave orthogonality will select from the sum only the component whose in-plane momentum $\mathbf{k}_{x y}+\mathbf{g}$ matches $\mathbf{K}_{x y}$ (corrected for the photon momentum $p=h v / \mathrm{c}$ ), i.e., $I_{\mathrm{PE}}\left(\mathbf{K}_{x y}\right) \propto\left|A_{\mathbf{K}_{x y}}\right|^{2}$. Therefore, the $\left(K_{x}, K_{y}\right)$-dependent ARPES maps in Fig. $2 \mathrm{a}-\mathrm{c}$ visualize essentially the $2 \mathrm{D}$ Fourier expansion of $\psi_{\mathrm{QWS}} 30,31$

We will now analyze $I_{\mathrm{PE}}$ as a function of photoelectron $K_{z}$ outof-plane momentum varied in the experiment through $h v$. The corresponding iso- $E_{\mathrm{B}}$ map in $\left(K_{x}, K_{z}\right)$ coordinates near the VBM is displayed in Fig. 3a. Its $K_{z}$-dispersive contours demonstrate the three-dimensional (3D) character of the VB states inherited from bulk GaN. The FS in Fig. 3b formed by the QWSs demonstrates a different behavior however: the ARPES signal sharply increases whenever $K_{z}$ approaches values of integer $G_{z}-$ corresponding to the $\Gamma$-points of the bulk BZ, but without any sign of $K_{z}$ dispersion. The latter is emphasized by the zooms in Fig. 3d,e, where the QWSs form segments straight in the $K_{z}$ direction. This pattern is characteristic of the $2 \mathrm{D}$ nature of the QWSs. The corresponding Fermi intensity is represented by $E_{\mathrm{F}^{-}}$ MDCs in Fig. $3 \mathrm{c}$ that show periodic oscillations peaked where $K_{z}$ matches the $\Gamma$-points.

Why do the QWSs display such an ARPES response, periodically oscillating as a function of $h v$ ? The dependence of $\psi_{\text {QWS }}$ on $z$ for given $\mathbf{K}_{x y}$ is represented as $\psi_{\text {QWS }}(z)=E(z) \cdot B_{k_{z}}(z)$, where the envelope function $E(z)$ confines the oscillating Bloch wave $B_{k_{z}}(z)$ whose $k_{z}$ momentum is adapted to the $3 \mathrm{D}$ crystal potential ${ }^{32}$. In our case, the first term is an Airy-like function embedded in the approximately triangular $V(z)$ and the second one derives from bulk states of the GaN host. Inheriting ideas of early ARPES studies on surface states ${ }^{32,33}$, it can be shown ${ }^{34}$ that if we expand $B_{k_{z}}(z)$ over out-of-plane reciprocal vectors $G_{z}$ of the $3 \mathrm{D}$ host lattice as $B_{k_{z}}(z)=\sum_{G_{z}} C_{G_{z}} e^{i\left(k_{z}+G_{z}\right) z}$, then the $K_{z}$ dependence of ARPES intensity for given $\mathbf{K}_{x y}$ appears as a sequence $I_{\mathrm{PE}}\left(K_{z}\right) \propto$ $\sum_{G_{z}}\left|C_{G_{z}}\right|^{2} P\left(K_{z}-\left(k_{z}+G_{z}\right)\right)$ of peaks $P\left(K_{z}-\left(k_{z}+G_{z}\right)\right)$ centered at $K_{\mathrm{z}}=k_{z}+G_{z}$. Physically, the ARPES intensity blows up whenever the photoelectron $K_{z}$ hits a $k_{z}+G_{z}$ harmonic of $\psi_{\text {QWS }}$ to maximize the $\langle f|$ and $|i\rangle$ scalar product. Amplitudes of the $I_{\mathrm{PE}}\left(K_{z}\right)$ peaks $\propto\left|C_{G_{z}}\right|^{2}$ image Fourier composition of the oscillating $B_{k_{z}}(z)$ term of $\psi_{\mathrm{QWS}}$ (modulated by $h v$-dependent photoelectron transmission through the AlGaN/AlN layer) and the peak shapes are related to the Fourier transform of the $E(z)$ term weighted by $e^{-\lambda_{\mathrm{PE}} z 34}$.

Importantly, the experimental $K_{z}$ dependence of the QWS signal in Fig. $3 \mathrm{~b}$ exhibits peaks exactly at $k_{z}+G_{z}$, corresponding to the $\Gamma$-point of bulk GaN. In combination with the $\left(K_{x}, K_{y}\right)$ image in Fig. 2, where the QWS signal corresponds to the same $\Gamma$ point, this fact confirms that the $\psi_{\mathrm{QWS}}$ 's are derived from the CBM states of bulk GaN. In a methodological perspective, such identification of the $\psi_{\text {QwS }}$ character can be essential, e.g., for heterostructures of layered transition metal dichalcogenides, where the $\mathrm{CB}$ can include two or more valleys almost degenerate in energy but separated in $\mathbf{k}$. The knowledge of the QWS character will then allow the predictive manipulation of the valley degree of freedom for potential valleytronics devices ${ }^{35}$.

We note that the common models of QWSs based on the 1D potential $V(z)$, such as that in Fig. 1b, imply that their in-plane behavior $\psi_{\text {QWS }}\left(\mathbf{r}_{x y}\right)$ is described by one single plane wave $e^{i \mathbf{K}_{x y} \boldsymbol{r}_{x y}}$ (i.e., one non-zero $A_{\mathbf{K}_{x y}}$ component) and out-of-plane behavior $\psi_{\mathrm{QWS}}(z)$ is identical to the smooth $E(z)$ function. However, the experimental FS maps in Fig. 2a,c reveal numerous non-zero $A_{\mathrm{K}}$ spread through $\mathbf{k}$-space and the $I_{P E}\left(K_{z}\right)$ oscillations numerous $k_{z}$ $+G_{z}$ harmonics. Accurate QWS models should therefore incorporate atomic corrugation of the interfacial potential in the in-plane and out-of-plane directions to form $\psi_{\mathrm{QWS}}$ as a confined Bloch wave.

The experimental distribution of high-energy ARPES intensity over sufficiently large volume of the $\left(K_{x}, K_{y}, K_{z}\right)$ space will in principle allow, notwithstanding the phase problem, a full reconstruction of $\Phi_{\mathrm{QWS}}(x, y, z)$ in all three spatial coordinates, 
a
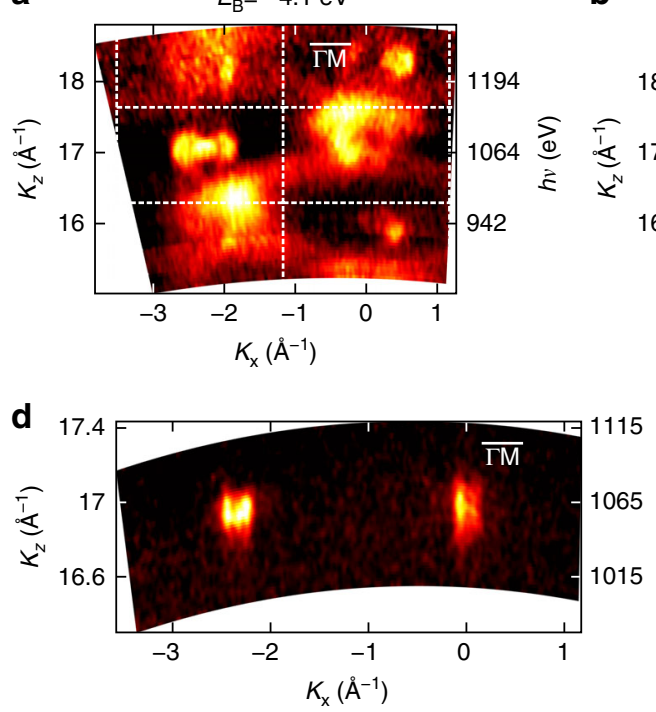

b
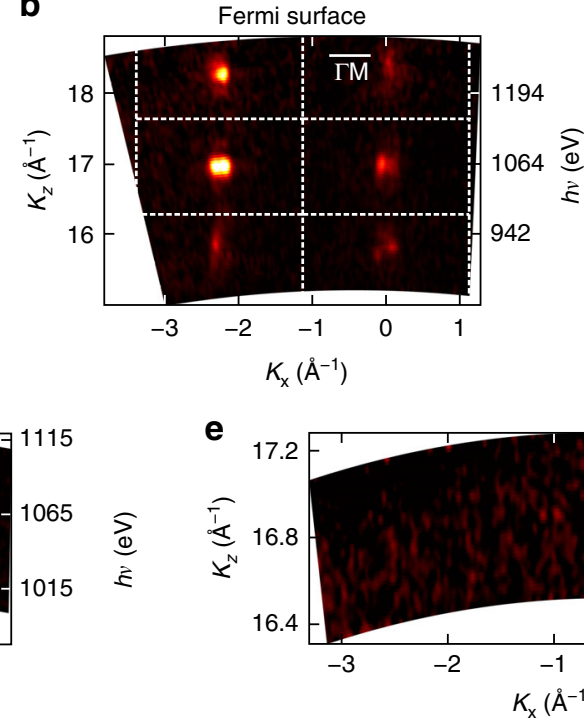

e

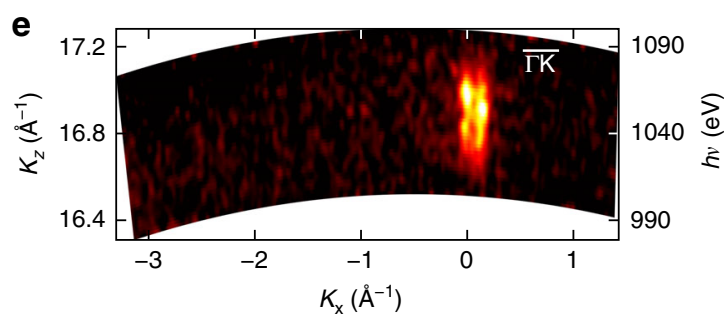

$\mathbf{C}$

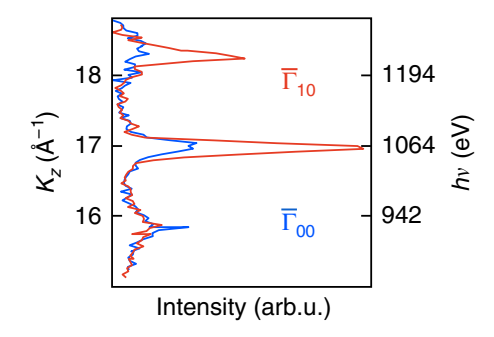

Max

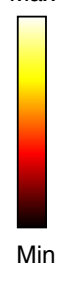

Fig. 3 ARPES response of the 2DEG as a function of $K_{z}$ momentum. ARPES intensity along the indicated azimuths is plotted as a function of $K_{z}$ rendered from $h v$ (the indicated $h v$ values correspond to the $\bar{\Gamma}$-point) a Iso- $E_{\mathrm{B}}$ map near the VBM, showing 3D contours of the VB states. b FS formed by the QWSs. c $E_{\mathrm{F}}-\mathrm{MDCS}$ at the $\bar{\Gamma}_{00}$ - and $\bar{\Gamma}_{10}$-points, showing periodically oscillating response of the QWSs. Its peaks located in the $\Gamma$-points evidence that the QWSs inherit their wavefunction from the CBM of parent bulk GaN. Zoom-in of the FS at the $\bar{\Gamma}_{00}$ - and $\bar{\Gamma}_{10}$-points along the $\overline{\Gamma M} \mathbf{d}$ and $\overline{\Gamma K} \mathbf{e}$ measured at around $1070 \mathrm{eV}$. The absence of its $K_{z}$ dispersion confirms the 2D character of the QWSs

similar to the reconstruction of molecular orbitals (see refs. 30,31, and references therein). This reconstruction will naturally incorporate full $\psi_{\text {OWS }}$ including the envelope and Bloch-wave terms that goes beyond the common $1 \mathrm{D}$ models such as in Fig. 1b, describing the QWSs as free 2D electrons with empirical $m^{\star}$ confined in the $z$ direction. More accurate models of the GaNHEMTs should replace free electrons by Bloch ones, naturally incorporating atomic corrugation.

Band dispersions: effective mass. Experimental band dispersions in GaN-HEMTs shown in Fig. 4 were measured along $\overline{\Gamma M}$ (a) and $\overline{\Gamma K}$ (b) at $h v=1066 \mathrm{eV}$ bringing $K_{z}$ to the $\Gamma$-point of the bulk BZ. Non-dispersive ARPES intensity coming from the AIN and AlGaN layers is suppressed in these plots by subtracting the angle-integrated spectral component. The CBM-derived QWSs appear as tiny electron pockets above the VB dispersions of GaN. Their energy separation from the VBM is consistent with the $\mathrm{GaN}$ fundamental band gap of $\sim 3.3 \mathrm{eV}$. Whereas the $\mathrm{VB}$ dispersions are broadened in $E_{\mathrm{B}}$ primarily because of band bending in the QW region, the QWS dispersions stay sharp. This confirms their 2D nature insensitive to band bending as well as their localization in the deep defect-free region in GaN, spatially separated from the defect-rich GaN/AlN interface region, the fundamental operational principle of the HEMTs delivering high $\mu_{\mathrm{e}}$. Fig. 4c,f,i show the experimental dispersions as a function of $k_{\mathrm{z}}$. Whereas clear dispersion of the VB states manifests their $3 \mathrm{D}$ character, the QWS are flat in $K_{z}$.

A zoom-in of the QWS dispersions along the $\overline{\Gamma M}$ and $\overline{\Gamma K}$ azimuths is shown in Fig. 4d,e with the corresponding MDC in Fig. 4g,h. Whereas the outer contour of these dispersions corresponds to the $\mathrm{QWS}_{1}$, the significant spectral weight in the middle is due to the $\mathrm{QWS}_{2}$. Following the $k_{\mathrm{F}}$ anisotropy discussed above, a parabolic fit of the QWS 1 dispersions yields $m^{*}$ values of $(0.16 \pm 0.03) m_{0}$ along the $\overline{\Gamma M}$ azimuths and $(0.13 \pm 0.02) m_{0}$ along $\overline{\Gamma K}\left(m_{0}\right.$ is the free-electron mass), which thus differ from each other by $\sim 22 \%$.

Our SX-ARPES experiment presents thus a direct evidence of the planar FS and $m^{*}$ anisotropy in GaN-HEMTs. This effect was overlooked in previous studies, because the optics methods are k- integrating and quantum oscillation techniques lose their $\mathbf{k}$ resolution in the $2 \mathrm{D}$ case. Magnetotransport experiments give only an indirect information on the 2DEG's $m^{* 10-12}$, which is conventionally ${ }^{36,37}$ assumed to be isotropic. We conjecture that further progress of SX-ARPES on energy resolution will push data analysis from merely band structure to one-electron spectral function $A(\omega, \mathbf{k})$, which will inform, e.g., the interaction of electrons with other quasiparticles such as the phonon-plasmoncoupled modes. ${ }^{38}$

Implications for the transport properties. How will the observed lateral anisotropy of the 2DEG electronic structure affect the transport properties? Naively, one might think that it would directly translate into an anisotropy of the electrical conductivity. However, fundamental linear response considerations attest that any physical properties such as conductivity described by a second-order tensor with $\mathrm{C}_{6 \mathrm{v}}$ symmetry must be scalar, i.e., in the linear (low-field) regime, conductivity in the hexagonal lattice of $\mathrm{GaN}$ must be isotropic (Supplementary Note 3). On the other hand, this restriction is lifted for the nonlinear (high-field) regime where conductivity can become anisotropic. A canonical example of such a crossover is $n$-doped Ge. ${ }^{39,40}$ Although its FS is anisotropic, cubic symmetry of the Ge lattice results in isotropic low-field conductivity. However, with an increase of the electric field, conductivity along the $\langle 001\rangle$, $\langle 011\rangle$, and $\langle 111\rangle$ crystallographic directions develops differently. The GaN-HEMTs can be easily driven into the nonlinear regime where electronic current saturates due to electron scattering on longitudinal optical (LO) phonons ${ }^{41,42}$. To reach the LO phonon energy, lighter electrons should gain a larger drift velocity $V_{\text {sat. }}$. Therefore, larger $V_{\text {sat }}$ and thus saturation current $I_{\text {sat }}$ should be expected in the directions of smaller $m^{*}$.

We have examined low- and high-field conductivity in our GaN-HEMT heterostructures using samples essentially identical to the ARPES ones, but with the $\mathrm{Al}_{0.45} \mathrm{Ga}_{0.55} \mathrm{~N}$ layer thickness increased to $15 \mathrm{~nm}$, to prevent a 2DEG degradation during longer sample handling in air. Hall measurements showed $n_{\mathrm{s}}=2 \times 10^{13} \mathrm{~cm}^{-2}, \mu_{\mathrm{e}}=1150 \mathrm{~cm}^{2} \mathrm{~V}^{-1} \mathrm{~s}$, and sheet resistance $R_{\mathrm{s}}$ $=240 \Omega \mathrm{sq}^{-1}$ for these samples. The fabricated test modules were oriented at four different angles $\left(0^{\circ}, 30^{\circ}, 60^{\circ}, 90^{\circ}\right)$ with respect to 

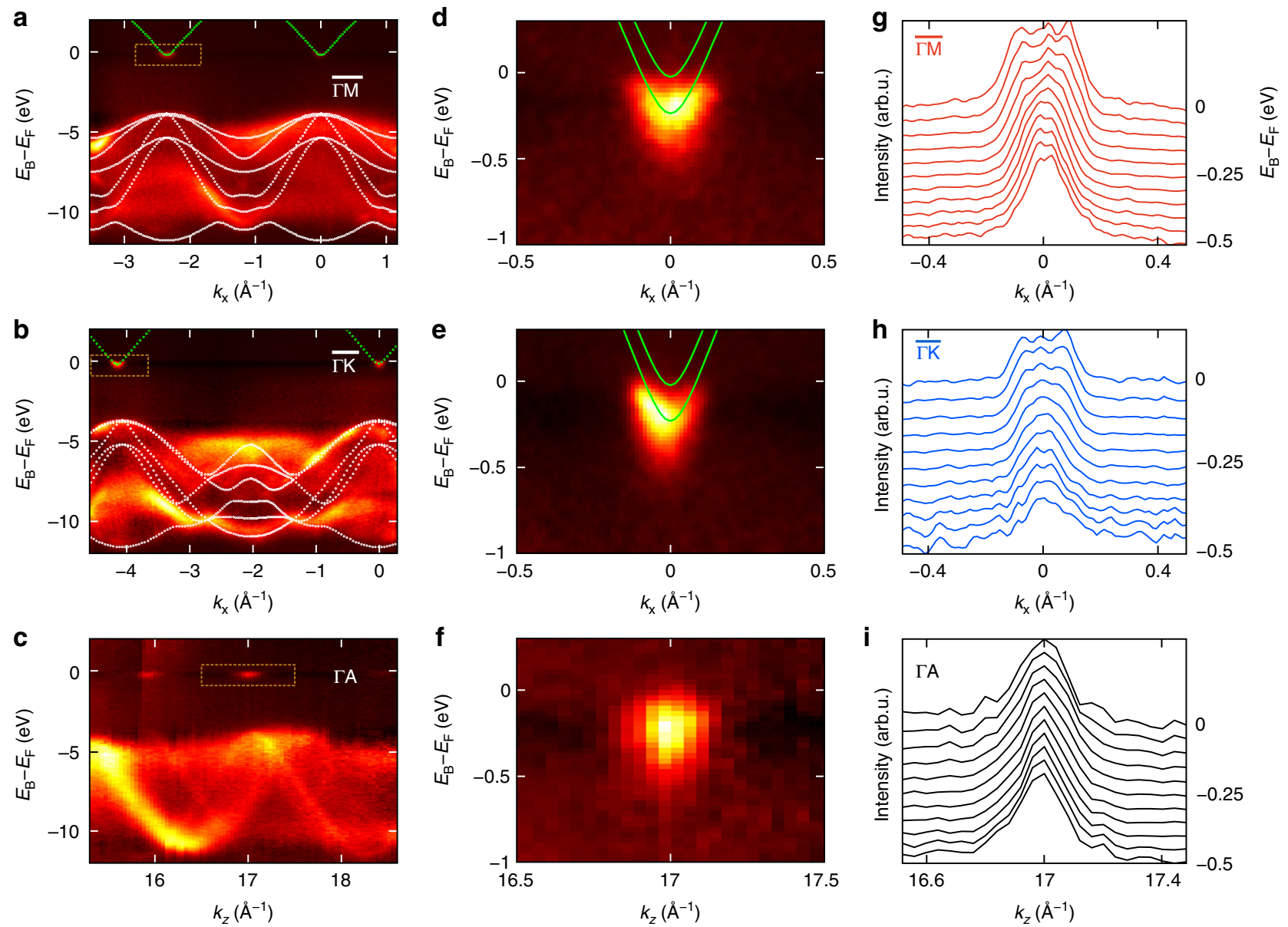

Min

Max

Fig. 4 Band dispersions of the buried 2DEG. a-c Experimental band structure measured at $h v=1066 \mathrm{eV}$ for the a $\Gamma K$ and $\mathbf{b} \Gamma M$ directions of the bulk $B Z$ (superimposed with calculated $E(\mathbf{k})$ of bulk $\mathrm{GaN}$, white dashed lines) and under variation of $h v$ for $\mathbf{c} \Gamma \mathrm{A}$, cf. Fig. 3. The CBM-derived QWSs appear above the VB continuum. $\mathbf{d}$-f Zoom-in image of the QWSs around the $\bar{\Gamma}_{10}$-point (green lines schematize their dispersions fitting the experimental $k_{F}$ ). $\mathbf{g}$-i (Normalized) MDCs around the $\bar{\Gamma}_{10}$-point for a series of $E_{\mathrm{B}}$ through the QWS bandwidth. The difference between the $\overline{\Gamma M}$ and $\overline{\Gamma K}$ dispersions manifests planar anisotropy of the 2DEG, and the absence of $k_{z}$ dispersion confirms its $2 \mathrm{D}$ character

a

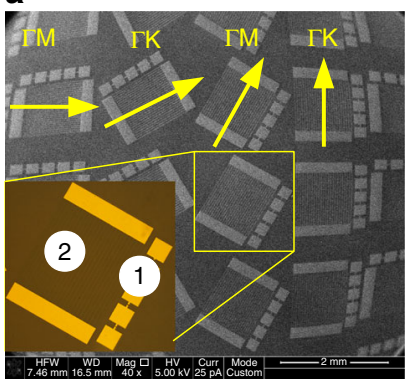

b

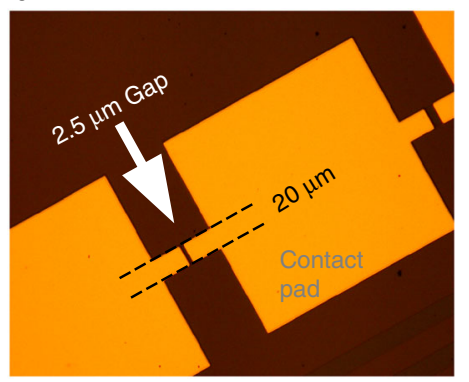

C

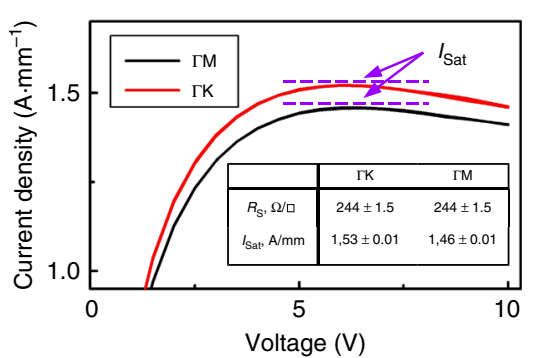

Fig. 5 Electron transport measurements. a Test modules, oriented in four directions (scanning electron microscope images, slightly distorted due to large view area): TLM modules for the contact resistance measurements (marked 1 in the inset) and 'resistor' modules for $R_{\mathrm{s}}$ determination (marked 2). The yellow arrows indicate the TLM azimuthal orientation. b Region of the TLM modules with the channel length $2.5 \mu \mathrm{m}$ used for the $I-V$ measurements (optical microscope image). c $l-V$ characteristics for the $\overline{\Gamma M}$ and $\overline{\Gamma K}$ azimuths. The inset table summarizes the mean $R_{\mathrm{s}}$ and $I_{\mathrm{sat}}$ values for different azimuths. Although $R_{\mathrm{s}}$ is essentially isotropic, higher $I_{\text {sat }}$ for the $\overline{\Gamma \mathrm{K}}$ azimuth in comparison with $\overline{\Gamma \mathrm{M}}$ indicates smaller $m^{\star}$ of electrons moving along $\overline{\Gamma \mathrm{K}}$

the substrate to promote current flow along the $\overline{\Gamma M}$ and $\overline{\Gamma K}$ azimuths (Fig. 5a,b). Results of the transport measurements presented in Fig. 5c show, as expected, isotropic low-field $R_{\mathrm{s}}$. However, the $I_{\text {sat }}$ characteristic of the high-field regime is clearly anisotropic: $1.53 \pm 0.01 \mathrm{~A} \times \mathrm{mm}^{-1}$ along $\overline{\Gamma K}$ and $1.46 \pm 0.01$
A $\times \mathrm{mm}^{-1}$ along $\overline{\Gamma M}$ (Fig. $5 \mathrm{c}$ ). As a consistency check, the modules rotated by $60^{\circ}$ with respect to each other showed the same $I_{\text {sat }}$ values, in accordance with hexagonal symmetry of the GaN electronic structure. These results on the previously overlooked $I_{\text {sat }}$ anisotropy demonstrate that $m^{*}$ along 
$\overline{\Gamma K}$ is smaller compared to $\overline{\Gamma M}$, as predicted by our ARPES results.

\section{Discussion}

Our direct $\mathbf{k}$-space imaging of the fundamental electronic structure characteristics-FS, band dispersions and occupancy, and Fourier composition of wavefunctions-of the 2DEG formed in high-frequency GaN-HEMTs with ultrathin barrier layer makes a quantitative step compared to conventional optics and magnetotransport experiments. We discover, in particular, significant planar anisotropy of the 2DEG band dispersions caused by piezoelectrically active relaxation of atomic position near the GaN/ AlN interface. This effect is found to manifest itself in nonlinear electron transport properties as anisotropy of the saturation drift velocity and current. Our findings suggest a positive use of the crystallographic orientation to improve these high-power characteristics of GaN-HEMTs. Furthermore, our k-space image of the Fourier composition of the 2DEG wavefunctions calls for extension of the conventional 1D models of semiconductor interfaces to 3D ones based on the Bloch-wave description naturally incorporating atomic corrugation. The fundamental knowledge about GaN-HEMTs achieved in our work as well as new device simulation methods can clarify the physical limits of these heterostructures, and finally push their reliable operation into the near- $\mathrm{THz}$ range. Methodologically, we have demonstrated the power of the synchrotron radiation based technique of SX-ARPES with its enhanced probing depth and sharp definition of the full $3 \mathrm{D} \mathbf{k}$ for the discovery of previously obscured properties of semiconductor heterostructures. Our results complement previous applications of SX-ARPES to buried oxide interfaces ${ }^{7}$ and magnetic impurities in semiconductors ${ }^{43}$ and topological insulators ${ }^{44}$, which used elemental and chemical-state specificity of this technique achieved with resonant photoemission. In a broader perspective, our methodology arms the heterostructure growth technology with means to directly control the fundamental $\mathbf{k}$-space parameters of the electronic structure, thereby delivering optimal transport and optical properties of the fabricated devices. Complementary to imaging of non-equilibrium electron motion in spatial coordinates, ${ }^{45}$ we can envisage an extension of our experimental methodology to pump-probe experiments using X-ray free-electron laser sources, which will image the electron system evolution in $\mathbf{k}$-space during transient processes in electronic devices.

\footnotetext{
Methods

Sample fabrication. The GaN-HEMT heterostructures embedding a 2DEG were grown on $c$-oriented sapphire substrate in a SemiTeq STE3N MBE-system equipped with an ammonia $\left(\mathrm{NH}_{3}\right)$ nitrogen source. The buffer layer growth adopted the procedure described in ref. 23. Before deposition, the substrate was annealed during $1 \mathrm{~h}$ and then nitrided for $40 \mathrm{~min}$ with $30 \mathrm{sccm} \mathrm{NH} \mathrm{NH}_{3}$ at $850{ }^{\circ} \mathrm{C}$. The following growth was carried out with $200 \mathrm{sccm} \mathrm{NH}$. Deposition started with 20 $\mathrm{nm}$ AlN layer, grown at $1050^{\circ} \mathrm{C}$. The following $200 \mathrm{~nm}$ AlN layer was grown at $1120^{\circ} \mathrm{C}$ with $\mathrm{Ga}$ used as a surfactant. Then a gradient junction to $\mathrm{Al}_{0.43} \mathrm{Ga}_{0.57} \mathrm{~N}$ with a thickness of $\sim 250 \mathrm{~nm}$ was achieved by a gradual decrease of the substrate temperature down to $T=830^{\circ} \mathrm{C}$, followed by $140 \mathrm{~nm}$ of growth at constant $T$. Next, a second gradient junction to $\mathrm{Al}_{0.1} \mathrm{Ga}_{0.9} \mathrm{~N}$ with a thickness of $\sim 140 \mathrm{~nm}$ was formed by reducing $T$ of the $\mathrm{Al}$ effusion cell. Then a $500 \mathrm{~nm} \mathrm{GaN}$ layer was grown. The growth was finished by deposition of a barrier layer consisting of $2 \mathrm{~nm} \mathrm{AlN}$ and $1 \mathrm{~nm} \mathrm{Al}_{0.5} \mathrm{Ga}_{0.5} \mathrm{~N}$ for the ARPES experiments, and $1 \mathrm{~nm}$ AlN and $15 \mathrm{~nm}$ $\mathrm{Al}_{0.45} \mathrm{Ga}_{0.55} \mathrm{~N}$ for measurements of transport properties. Hall effect characterization was carried out in magnetic fields up to $40 \mathrm{kG}$. The magnetic-field dependences were measured in both the standard Hall and van der Pauw geometries. All transport measurements were carried out at the Resource Center of Electrophysical Methods (Complex of NBICS-Technologies of Kurchatov Institute).

SX-ARPES experiments. Raw SX-ARPES data were generated at the Swiss Light Source synchrotron radiation facility (Paul Scherrer Institute, Switzerland). The experiments have been carried out at the SX-ARPES endstation ${ }^{46}$ of the ADRESS beamline ${ }^{47}$, delivering high photon fluxes up to $10^{13}$ photons $\times \mathrm{s}^{-1} \times(0.01 \% \mathrm{BW})^{-1}$. With the actual experimental geometry, $p$-polarized incident $\mathrm{X}$-rays selected
}

electron states symmetric relative to the $\overline{\Gamma M}$ and $\overline{\Gamma K}$ azimuths. The projection $K_{x}$ of the photoelectron momentum was directly measured through the emission angle along the analyser slit, $K_{y}$ is varied through tilt rotation of the sample, and $k_{z}$ through $h v$. The experiments were carried out at $12 \mathrm{~K}$ to quench the thermal effects reducing the coherent $\mathbf{k}$-resolved spectral component at high photoelectron energies ${ }^{48}$. The combined (beamline and analyzer) energy resolution was $\sim 150$ $\mathrm{meV}$ and the angular resolution of the PHOIBOS-150 analyzer was $0.07^{\circ}$. The $\mathrm{X}$ ray spot size in projection on the sample was $30 \times 75 \mu \mathrm{m}^{2}$, which allowed us to control spatial homogeneity of our samples. Charging effects were not detected due to the small thickness of the AlGaN barrier layer.

Electronic structure calculations. First-principles calculations for bulk GaN have been carried out in the DFT framework as implemented in the pseudopotential Quantum Espresso code ${ }^{49}$ using ultrasoft pseudopotentials. The electron exchangecorrelation term was treated within the Generalized Gradient Approximation using the Perdew-Burke-Ernzerhof functional. Self-consistent calculations for bulk GaN were performed with the plane-wave cutoff energy $60 \mathrm{Ry}$ and $\mathbf{k}$-space sampling over a grid of $10 \times 10 \times 5$ points in the $\mathrm{BZ}$ and corrected with the scissors operator to reproduce the experimental band gap. Calculations for the GaN-HEMT heterostructure used a $1 \times 1$ slab geometry with the supercell including 18 u.c. of GaN in the middle between 3 u.c of AlN at each end, Fig. $2 \mathrm{~g}$. Atomic coordinates in the supercell were relaxed, but imposing the lateral u.c. of bulk $\mathrm{GaN}$ until the Hellmann-Feynman forces on each atom were $<30 \mathrm{meV}^{-1}$. The plane-wave cutoff energy was $25 \mathrm{Ry}$ with a k-grid of $10 \times 10 \times 1$ points. The Gaussian window for LDOS calculations was set to $0.05 \mathrm{eV}$.

Transport measurements. Raw transport data were generated at the Kurchatov Institute. To measure linear and nonlinear transport properties of the GaN-HEMT heterostructures, two types of test modules with low-resistance regrown ohmic contacts $^{50,51}$ were formed. Details on processing can be found in ref. 51. The first type modules were conventional transmission line measurement (TLM) modules with a channel width of $20 \mu \mathrm{m}$ and channel lengths of $2.5,10,20$, and $40 \mu \mathrm{m}$ (marked 1 in Fig. 5a). These modules were used to determine contact resistance, which was found to be $0.15 \Omega \mathrm{mm}$. Also $I-V$ curves were measured at the smallest gaps $(2.5 \mu \mathrm{m}$ length channels, see Fig. 5b) of 24 such TLM modules (6 modules per each of four directions) in DC mode. The voltage sweep time (1 ms per point with a voltage step of $0.5 \mathrm{~V}$ ) was chosen to be small enough to suppress sample heating effects as judged by the absence of hysteresis in the forward and backward voltage scans as well as repeatability of the $I-V$ curves with a sweep time reduction. The second type "resistor" modules (marked 2 in Fig. 5a) were arrays of $1 \mathrm{~mm}$ long and $20 \mu \mathrm{m}$ wide stripes ( 25 stripes per module separated by $20 \mu \mathrm{m}$ mesa isolation) with contact pads on each side. These modules had negligible contact resistance and were used for precise measurement of the 2 DEG low-field conductivity in different directions.

Data availability. Derived data supporting the findings of this study are available from the corresponding author on request. The SX-ARPES data were processed using the custom package MATools available at https://www.psi.ch/sls/adress/manuals.

Received: 15 August 2017 Accepted: 9 April 2018

Published online: 11 July 2018

\section{References}

1. Mimura, T. The early history of the high electron mobility transistor (HEMT). IEEE Trans. Microw. Theory Techn. 50, 780-782 (2002).

2. Gurusinghe, M. N., Davidsson, S. K. \& Andersson, T. G. Two-dimensional electron mobility limitation mechanisms in $\mathrm{Al}_{\mathrm{x}} \mathrm{Ga}_{1-\mathrm{x}} \mathrm{N} / \mathrm{GaN}$ heterostructures. Phys. Rev. B 72, 045316 (2005).

3. Medjdoub, F. \& Iniewski, K. (eds). Gallium Nitride (GaN): Physics, Devices, and Technology (CRC Press, Taylor \& Francis Group, Boca Raton, 2016).

4. Yu, E. T. et al. Measurement of piezoelectrically induced charge in GaN/ AlGaN heterostructure field-effect transistors. Appl. Phys. Lett. 71, 2794-2796 (1997).

5. Gaska, R. et al. The influence of the deformation on the two-dimensional electron gas density in GaN-AlGaN heterostructures. Appl. Phys. Lett. 72, 64-66 (1998).

6. Tsukazaki, A., Ohtomo, A. \& Kita, T. Quantum Hall effect in polar oxide heterostructures. Science 315, 1388-1391 (2007).

7. Cancellieri, C. et al. Polaronic metal state at the $\mathrm{LaAlO}_{3} / \mathrm{SrTiO}_{3}$ interface. Nat Commun. 7, 10386 (2016)

8. Chen, Y. et al. Creation of high mobility two-dimensional electron gases via strain induced polarization at an otherwise nonpolar complex oxide interface. Nano Lett. 15, 1849-1854 (2015).

9. Nagatsuma, T., Ducournau, G. \& Renaud, C. C. Advances in terahertz communications accelerated by photonics. Nat. Photonics 10, 371-379 (2016).

10. Shockley, W. Cyclotron resonances, magnetoresistance, and Brillouin zones in semiconductors. Phys. Rev. 90, 491 (1953). 
11. Krieger, J. B., Meeks, T. \& Esposito, E. Conductivity in anisotropic semiconductors: application to longitudinal resistivity and Hall effect in saturation-stressed degenerately doped $n$-type germanium. Phys. Rev. B 5 1499-1504 (1972).

12. Brandt, N. B. \& Chudinov, S. M. Shubnikov-de Haas effect and its application to investigation of the energy spectrum of metals, semimetals, and semiconductors. Sov. Phys. Usp. 25, 518-529 (1982).

13. Strocov, V. N. et al. Soft-X-ray ARPES at the Swiss Light Source: From 3D materials to buried interfaces and impurities. Synchrotron Radiat. News 27, 31-40 (2014).

14. Powell, C. J., Jablonski, A., Tilinin, I. S., Tanuma, S. \& Penn, D. R. Surface sensitivity of Auger-electron spectroscopy and X-ray photoelectron spectroscopy. J. Electron Spectrosc. Relat. Phenom. 98/99, 1-15 (1999).

15. Gray, A. X. et al. Bulk electronic structure of the dilute magnetic semiconductor $\mathrm{Ga}_{1-\mathrm{x}} \mathrm{Mn}_{\mathrm{X}} \mathrm{As}$ through hard X-ray angle-resolved photoemission. Nat. Mater. 11, 957-962 (2012).

16. Krawczyk, M., Zommer, L., Jablonski, A., Grzegory, I. \& Bockowski, M. Energy dependence of electron inelastic mean free paths in bulk GaN crystals. Surf. Sci. 566-568, 1234-1239 (2004).

17. Strocov, V. N. Intrinsic accuracy in 3-dimensional photoemission band mapping. J. Electron Spectros. Relat. Phenom. 130, 65-78 (2003).

18. Strocov, V. N. et al. Three-dimensional electron realm in $\mathrm{VSe}_{2}$ by soft-X-ray photoelectron spectroscopy: origin of charge-density waves. Phys. Rev. Lett. 109, 086401 (2012).

19. Yeh, J. J. \& Lindau, I. Atomic subshell photoionization cross sections and asymmetry parameters: $1 \leq \mathrm{Z} \leq 103$. At. data Nucl. data Tables 32, 1-155 (1985).

20. Cao, Y. et al. MBE growth of high conductivity single and multiple AlN/GaN heterojunctions. J. Cryst. Growth 323, 529-533 (2011).

21. Kaun, S. W. et al. Pure AlN layers in metal-polar AlGaN/AlN/GaN and AlN/ $\mathrm{GaN}$ heterostructures grown by low-temperature ammonia-based molecular beam epitaxy. Semicond. Sci. Technol. 30, 055010 (2015).

22. Guo, L. et al. Hot electron induced non-saturation current behavior at high electric field in InAlN/GaN heterostructures with ultrathin barrier. Sci. Rep. 6, 37415 (2016).

23. Mayboroda, I. et al. Growth of AlGaN under the conditions of significant gallium evaporation: phase separation and enhanced lateral growth. J. Appl. Phys. 122, 105305 (2017).

24. Lu, F. et al. Magneto-transport spectroscopy of the first and second twodimensional subbands in $\mathrm{Al}_{0.25} \mathrm{Ga}_{0.75} \mathrm{~N} / \mathrm{GaN}$ quantum point contacts. Sci. Rep. 7, 42974 (2017).

25. Straub, Th et al. Many-body definition of a Fermi surface: application to angle-resolved photoemission. Phys. Rev. B 55, 13473-13478 (1997).

26. Svane, A. et al. Quasiparticle self-consistent GW theory of III-V nitride semiconductors: bands, gap bowing, and effective masses. Phys. Rev. B 82, $115102(2010)$

27. Luttinger, J. M. Fermi surface and some simple equilibrium properties of a system of interacting fermions. Phys. Rev. 119, 1153-1163 (1960).

28. Mishra, M. K. et al. Comprehensive magnetotransport characterization of two dimensional electron gas in $\mathrm{AlGaN} / \mathrm{GaN}$ high electron mobility transistor structures leading to the assessment of interface roughness. AIP Adv. 4, 097124 (2014)

29. Feibelman, P. J. \& Eastman, D. E. Photoemission spectroscopy correspondence between quantum theory and experimental phenomenology. Phys. Rev. B 10, 4932-4947 (1974).

30. Puschnig, P. et al. Reconstruction of molecular orbital densities from photoemission data. Science 326, 702-706 (2009).

31. Kliuiev, P., Latychevskaia, T., Osterwalder, J., Hengsberger, M. \& Castiglioni, L. Application of iterative phase-retrieval algorithms to ARPES orbital tomography. New. J. Phys. 18, 093041 (2016).

32. Ortega, J. E., Himpsel, F. J., Mankey, G. J. \& Willis, R. F. Quantum-well states and magnetic coupling between ferromagnets through a noble-metal layer. Phys. Rev. B 47, 1540-1552 (1993).

33. Louie, S. G. et al. Periodic oscillations of the frequency-dependent photoelectric cross sections of surface states: theory and experiment. Phys. Rev. Lett. 44, 549-553 (1980).

34. Strocov, V. N. Photoemission response of 2D states. Preprint at http://arXiv. org/abs/1801.07505 (2018).

35. Schaibley, J. R. et al. Valleytronics in 2D materials. Nat. Rev. Mater. 1, 16055 (2016).

36. Knap, W. et al. Effective g $^{*}$ factor of two-dimensional electrons in GaN/ AlGaN heterojunctions. Appl. Phys. Lett. 75, 3156-3158 (1999).

37. Syed, S. et al. Nonparabolicity of the conduction band of wurtzite GaN. Appl. Phys. Lett. 83, 4553-4555 (2003).

38. Talwar, D. N. Direct evidence of LO phonon-plasmon coupled modes in nGaN. Appl. Phys. Lett. 97, 051902 (2010).

39. Schweitzer, D. \& Seeger, K. The anisotropy of conductivity of n-type germanium in strong D.C. fields. Z. für Phys. 183, 207-216 (1965).

40. Nathan, M. I. Anisotropy of the conductivity of $n$-type germanium at high electric fields. Phys. Rev. 130, 2201-2204 (1963).
41. Fang, T. et al. Effect of optical phonon scattering on the performance of GaN transistors. IEEE Electron Dev. Lett. 33, 709-711 (2012).

42. Gonschorek, M. et al. Self heating in AlInN/AlN/GaN high power devices: origin and impact on contact breakdown and IV characteristics. J. Appl. Phys. 109, 063720 (2011).

43. Kobayashi, M. et al. Unveiling the impurity band induced ferromagnetism in the magnetic semiconductor (Ga,Mn)As. Phys. Rev. B 89, 205204 (2014).

44. Krieger, J. A. et al. Spectroscopic perspective on the interplay between electronic and magnetic properties of magnetically doped topological insulators. Phys. Rev. B 96, 184402 (2017).

45. Man, M. K. L. et al. Imaging the motion of electrons across semiconductor heterojunctions. Nat. Nanotech. 12, 36-40 (2017)

46. Strocov, V. N. et al. Soft-X-ray ARPES facility at the ADRESS beamline of the SLS: concepts, technical realisation and scientific applications. J. Synchrotron Rad. 21, 32-44 (2014).

47. Strocov, V. N. et al. High-resolution soft X-ray beamline ADRESS at the Swiss Light Source for resonant inelastic X-ray scattering and angle-resolved photoelectron spectroscopies. J. Synchrotron Rad. 17, 631-643 (2010).

48. Braun, J. et al. Exploring the XPS limit in soft and hard $\mathrm{x}$-ray angle-resolved photoemission using a temperature-dependent one-step theory. Phys. Rev. B 88, 205409 (2013)

49. Giannozzi, P. et al. Quantum expresso: a modular and open-source software project for quantum simulations of materials. J. Phys. Condens. Matter 21, 395502 (2009).

50. Yue, Y. et al. InAlN/AlN/GaN HEMTs with regrown ohmic contacts and $f_{T}$ of 370 GHz. IEEE Electron Dev. Lett. 33, 988-990 (2012).

51. Mayboroda, I. et al. Selective MBE growth of nonalloyed ohmic contacts to $2 \mathrm{D}$ electron gas in high-electron-mobility transistors based on GaN/AlGaN heterojunctions. Tech. Phys. Lett. 40, 488-490 (2014).

\section{Acknowledgements}

We thank M.B. Tsetlin, V.G. Nazin, E.E. Krasovskii, and G. Aeppli for fruitful discussions. M.-A.H. was supported by the Swiss Excellence Scholarship grant ESKAS-no. 2015.0257. N.K.C. was partly supported by the Grant RFBR 16-07-01188.

\section{Author contributions}

L.L.L. and V.N.S. conceived the SX-ARPES research at the Swiss Light Source. L.L.L., M.A.H., and V.N.S. performed the SX-ARPES experiment supported by X.W., B.T., and T.S. L.L.L. and V.N.S. processed and interpreted the data. V.N.S. set theoretical description of the ARPES response. M.-A.H. performed the DFT calculations. M.L.Z., I.O.M., E.S.G., and V.G.V. conceived the GaN-HEMT project at the Kurchatov Institute. I.O.M. and I.S E. grew the samples supported by M.L.Z. N.K.C. performed Hall characterization. E.S.G. implemented the 2DEG numerical model. I.O.M., I.A.C., and M.L.Z. fabricated the TLM modules and performed transport measurements. V.N.S. wrote the manuscript with contributions of L.L.L., V.G.V., E.S.G., and I.O.M. All authors discussed the results, interpretations, and scientific concepts.

\section{Additional information}

Supplementary Information accompanies this paper at https://doi.org/10.1038/s41467018-04354-x.

Competing interests: The authors declare no competing interests.

Reprints and permission information is available online at http://npg.nature.com/ reprintsandpermissions/

Publisher's note: Springer Nature remains neutral with regard to jurisdictional claims in published maps and institutional affiliations.

cc (i) Open Access This article is licensed under a Creative Common Attribution 4.0 International License, which permits use, sharing, adaptation, distribution and reproduction in any medium or format, as long as you give appropriate credit to the original author(s) and the source, provide a link to the Creative Commons license, and indicate if changes were made. The images or other third party material in this article are included in the article's Creative Commons license, unless indicated otherwise in a credit line to the material. If material is not included in the article's Creative Commons license and your intended use is not permitted by statutory regulation or exceeds the permitted use, you will need to obtain permission directly from the copyright holder. To view a copy of this license, visit http://creativecommons.org/ licenses/by/4.0/.

(c) The Author(s) 2018 